\title{
HIV Bio-behavioral Risk Study Implementation in Resource-poor Military Settings
}

\author{
Stacy M. Endres-Dighe*, Lauren Courtney and Tonya Farris
}

Biostatistics and Epidemiology Division, RTI International, Research Triangle Park, NC, USA

\section{Objective}

We present lessons learned from over a decade of HIV biobehavioral risk study implementation and capacity-building in African militaries.

\section{Introduction}

Circumstances within the military environment may place military personnel at increased risk of contracting sexually transmitted infections (STI) including HIV. HIV bio-behavioral risk studies provide a critical source of data to estimate HIV/STI prevalence and identify risk factors, allowing programs to maximize impact by focusing on the drivers of the epidemic.

\section{Methods}

Since 2005, RTI has provided technical assistance (TA) to support HIV/STI Seroprevalence and Behavioral Epidemiology Risk Surveys (SABERS) in 14 countries across Sub-Saharan Africa and Asia. SABERS are cross-sectional studies consisting of a survey to assess knowledge, attitudes and behaviors related to HIV, coupled with rapid testing for HIV and other STIs. RTI tailored each survey instrument to be culturally appropriate in content and methodology, trained military personal to serve as data collection staff, and provided logistical support for study implementation.

\section{Results}

Key lessons learned are summarized below:

Data collection mode varied from paper-based to computerassisted surveys, depending on country preference, in-country staff capabilities, and the country's technological capacity. Computerassisted data collection systems were preferable because they improved data quality through the use of programmed skip patterns, range, and consistency checks. By eliminating the need for data entry, computer-assisted systems also saved program resources and enabled faster access to the data for analysis.

Survey administration method varied from self-administered to interviewer-administered surveys. Literacy rates, technological familiarity, and confidentiality concerns were key drivers in determining the best data collection method. Self-administered surveys such as computer-assisted self-interview (CASI) were preferable due to the high-level of confidentiality they provide, but required a high-level of literacy and computer familiarity. If confidentiality was a big concern in low-literacy settings, audio computer-assisted self-interview (ACASI) was used if the population had some computer familiarity. Interviewer-administered surveys such as computer-assisted personal interview (CAPI) were used in most low-literacy settings.

Tailoring the survey instrument and administration for cultural appropriateness was vital to the acquisition of sound, viable data. Sexual behaviors and the definition of "regular sexual partner" and other terms varied according to local custom. The sensitive nature of the survey questions also impacted survey administration operationally. The preference for same-sex or opposite sex interviewers varied by country and military setting. It was imperative to pre-test the survey.
A skilled workforce and staff retention are essential to provide high quality data. Literacy levels, technological familiarity, HIV knowledge, and time commitments must all be considered when selecting data collection staff. Retention of staff throughout the duration of data collection activities can be a major issue especially among military personnel who were often called away from study activities to perform military duties.

Host military ownership was integral to the success of the SABERS program. By engaging military leadership early and involving them in all decision making processes we ensured the partner military was invested in the study and its success and found value in the resulting data and findings. Host militaries were actively involved in SABERS by providing staff for data collection, leading sensitization activities, and monitoring data collection activities in the field.

Inclusion of capacity building elements during study implementation led to increased host military buy-in. Capacity building included staff trainings and practical experience in survey methodology, use of electronic data collection instruments, study logistics and data monitoring.

Confidentiality of survey data and HIV test results was of increased concern given that these studies were conducted in a work place environment. For this reason, it was imperative to assure participants that disclosures of drug or alcohol use and positive HIV/STI test results would remain confidential and would not affect their military employment.

\section{Conclusions}

Based on our experience, the following are required for the successful implementation of an HIV Bio-behavioral Risk Study in resource-poor military settings: (1) selection of a data collection mode and survey administration method that is context-appropriate, (2) utilization of local wording and customs, (3) a skilled workforce, (4) local buy-in/partnership, (5) inclusion of capacity building elements, and (6) assurance of confidentiality.

\section{Keywords}

survey; capacity building; militaries; resource poor; Africa

\section{*Stacy M. Endres-Dighe}

E-mail: sendres@rti.org 